\title{
The role of autolysins during vegetative growth of Bacillus subtilis 168
}

\author{
Steve A. Blackman, Thomas J. Smith and Simon J. Foster
}

Department of Molecular Biology and

Biotechnology, University of Sheffield, Firth Court, Western Bank, Sheffield S10 2TN, UK
Author for correspondence: Simon J. Foster. Tel: +44 1142224411 . Fax: +44 1142728697. e-mail: s.foster@sheffield.ac.uk

\begin{abstract}
A set of isogenic mutants of Bacillus subtilis 168, insertionally inactivated in the genes encoding a number of lytic enzymes and a sigma factor $\left(\sigma^{D}\right.$, which controls the expression of a number of autolysins) was constructed. Phenotypic analysis of the mutants determined the individual and combined roles of the autolysins in vegetative growth. The major vegetative autolysins of B. subtilis, LytC (50 kDa amidase) and LytD (90 kDa glucosaminidase), were shown to have roles in cell separation, cell wall turnover, antibiotic-induced lysis and motility. LytC was also shown to have a role in general cell lysis induced by sodium azide. Renaturing SDS-PAGE of cell-wall-binding protein extracts of the mutant strains revealed the presence of a novel autolysin that was previously masked by LytC. This $\mathbf{4 9} \mathrm{kDa}$ enzyme was shown to be $\sigma^{D}$. controlled and was identified as a candidate cell separation and cell wall turnover enzyme. A multiple mutant strain, lacking LytC, LytD and the 49 kDa enzyme, retained at least ten bands of autolytic activity. These may correspond to individual or proteolytically processed novel autolysins, the functions of which are unknown. The multiple mutant strains facilitate the study of these, and other lytic enzymes, to determine their cellular functions.
\end{abstract}

Keywords: Bacillus subtilis, autolysins, cell separation, cell wall turnover, motility

\section{INTRODUCTION}

Seemingly, all bacteria possess a complement of autolysins, which are potentially lethal enzymes capable of hydrolysing peptidoglycan, the major structural component of bacterial cell walls (Ghuysen et al., 1966). These enzymes have been postulated to have roles in motility, cell separation, competence, antibiotic-induced lysis, pathogenicity, cell wall growth and turnover, and differentiation (Rogers et al., 1983; Ward \& Williamson, 1984). Bacillus subtilis has multiple autolysins and associated prophage lytic enzymes, the proposed functions of which were summarized by Smith et al. (1996).

The two major autolysins present during vegetative growth of $B$. subtilis are a $50 \mathrm{kDa} \mathrm{N}$-acetylmuramoyl-Lalanine amidase (amidase) and a $90 \mathrm{kDa}$ endo- $\beta-N$ acetylglucosaminidase (glucosaminidase), which have been purified and characterized (Herbold \& Glaser, 1975; Rogers et al., 1984). Recently, their structural genes have been cloned and studied at the molecular level. The amidase is encoded by the lytC gene, which is

Abbreviation: CWBP(s), cell-wall-binding protein(s). part of a four-gene divergon, lytRABC (Kuroda \& Sekiguchi, 1991; Lazarevic et al., 1992; Kuroda et al., 1992). The lytR gene is transcribed divergently from the lyt $A B C$ operon and encodes a putative DNA-binding protein, which represses expression of itself and lyt $A B C$ (Lazarevic et al., 1992). The lytA gene encodes a putative lipoprotein (Lazarevic et al., 1992; Kuroda et al., 1992) and $l y t B$ encodes a modifier protein which enhances the activity of the amidase (Herbold \& Glaser, 1975; Lazarevic et al., 1992; Kuroda \& Sekiguchi, 1993). Transcription of this operon proceeds from two promoters. One is controlled by $\sigma^{\mathrm{D}}$, the sigma factor responsible for transcription of the genes involved in flagellar motility and chemotaxis (Helmann et al., 1988), which accounts for $70-90 \%$ of the transcription during vegetative growth. The other promoter upstream of lyt $A B C$ is controlled by $\sigma^{\mathrm{A}}$, the major house-keeping sigma factor of $B$. subtilis (Lazarevic et al., 1992; Kuroda \& Sekiguchi, 1993). The glucosaminidase is encoded by the lytD gene (Margot et al., 1994; Rashid et al., 1995) and its active form is a dimer of about $190 \mathrm{kDa}$ (Rogers et al., 1984). Transcription of lytD proceeds from a single promoter which is controlled by $\sigma^{\mathrm{D}}$ (Margot et al., 1994), although about $5 \%$ of transcription may be 
controlled by another minor promoter activity (Rashid et al., 1995). The genes encoding LytC and LytD have been insertionally inactivated both singly (Kuroda \& Sekiguchi, 1991; Margot \& Karamata 1992) and together (Rashid et al., 1993; Margot et al., 1994). Despite this, the specific functions of these enzymes have not been fully elucidated. LytC has a role in cell wall turnover, motility and cell lysis (Margot \& Karamata, 1992). It also has a mutually compensatory role with CwlC (a $30 \mathrm{kDa}$ sporulation-specific amidase) in mother cell lysis at the end of sporulation (Smith \& Foster, 1995). A lytC lytD double mutant has been shown to have reduced motility, however single mutants in lytC or lytD were unaffected (Rashid et al., 1993). It is surprising that the lytC lytD strain did not show abnormal filamentation under the conditions used as it has been previously postulated that one or both of the major autolysins has a role in cell separation (Fein $\&$ Rogers, 1976). This is exemplified by the fact that when the structural gene for SigD, which to a great extent controls the expression of both autolysins, is inactivated the mutant is filamentous (Helmann et al., 1988). A sigD mutant is still able to undergo septation but is filamentous owing to the lack of hydrolysis of the nascent septa, preventing daughter cell separation.

Most autolysins may perform more than one role in the cell and can also often compensate for the lack of each other (Foster, 1991). As a result of the possible functional redundancy of the autolysins of $B$. subtilis, it is important to identify and characterize the total complement of such enzymes to determine their individual and combined roles in any given cellular process. This study is ultimately concerned with identifying and characterizing novel autolysins which are expressed during, and so probably involved in, vegetative growth of $B$. subtilis. As part of this work multiple peptidoglycan hydrolase mutants of $B$. subtilis were constructed to facilitate the study of the role of the autolysins.

\section{METHODS}

Bacterial strains, plasmids and growth conditions. Strains of B. subtilis and plasmids used in this study are shown in Table 1. Unless otherwise stated, growth of $B$. subtilis strains was carried out at $37^{\circ} \mathrm{C}$ in nutrient broth (Oxoid) with shaking (250 r.p.m.) or on nutrient agar plates at $37^{\circ} \mathrm{C}$. Minimal medium was as described by Anagnostopoulos \& Spizizen (1961), supplemented, where appropriate, with amino acids $\left(50 \mu \mathrm{g}\right.$ tryptophan $\mathrm{ml}^{-1}, 220 \mu \mathrm{g}$ methionine $\left.\mathrm{ml}^{-1}\right)$. Chromosomal drug resistance markers in B. subtilis were selected with $1 \mu \mathrm{g}$ erythromycin $\mathrm{ml}^{-1}, 25 \mu \mathrm{g}$ lincomycin $\mathrm{ml}^{-1}, 5 \mu \mathrm{g}$ chloramphenicol ml $\mathrm{m}^{-1}, 0.3 \mu \mathrm{g}$ phleomycin $\mathrm{ml}^{-1}$, and $100 \mu \mathrm{g}$ spectinomycin $\mathrm{ml}^{-1}$. Spores of $B$. subtilis were prepared by growing in CCY medium (Stewart et al., 1981) for $2 \mathrm{~d}$ at $37^{\circ} \mathrm{C}$. Escherichia coli XLOLR (Stratagene) was grown in LuriaBertani broth or on Luria-Bertani plates at $37^{\circ} \mathrm{C}$. In E. coli, plasmids were selected with $50 \mu \mathrm{g}$ ampicillin $\mathrm{ml}^{-1}$.

\section{Construction of mutants}

lytC. Plasmid p6302 (Lazarevic et al., 1992) was restricted with $B g l I I$, which cuts once, within the cloned B. subtilis lyt $C$ gene. The linearized plasmid was end-filled and dephosphorylated, and ligated with a gel-purified $1.5 \mathrm{~kb}$ phleomycin resistance cassette that had been excised from plasmid pIC22 (Steinmetz \& Richter, 1994) by restriction with SmaI and PvuII. Restriction analysis of the resultant plasmid, pTJS43, using $B a m H I$ showed that the phleomycin resistance cassette had been inserted into the $l y t C$ gene and was oriented in the same direction as the $l y t C$ coding sequence. The lytC-inactivated strain of B. subtilis (SH125) was made by transforming (Anagnostopoulos \& Spizizen, 1961) B. subtilis 168 HR with pTJS43 (linearized by EcoRI digestion). Recombinants were selected by overlaying agar plates with top agar containing phleomycin $\left(0.3 \mu \mathrm{g} \mathrm{m}^{-1}\right)$, as described by Cutting \& Vander Horn (1990). Southern blot analysis showed that the phleomycin resistance cassette had been transferred from pTJS43 into the chromosomal copy of $l y t C$ by a double cross-over event to create strain $\mathrm{SH} 125$. The mutation was transferred into the parental background for this study (1A304) by transformation with SH125 genomic DNA.

lytD. The $1 \cdot 1 \mathrm{~kb}$ PstI fragment, containing part of the lytD gene, from p5102 (Margot et al., 1994) was ligated into the PstI restriction site of pUBS1 (from G. Murphy, Institute of Plant Science Research, Cambridge, UK) to create plasmid pSAB100. The spectinomycin resistance cassette was excised from pIC156 (Steinmetz \& Richter, 1994) using SmaI and ligated into the HpaI site of pSAB100 to create pSAB101. Restriction analysis of pSAB101, using $B a m \mathrm{HI}$, showed that the spectinomycin resistance cassette had been inserted into the $l y t D$ gene and was oriented in the opposite direction to the lytD coding sequence. Plasmid pSAB101 was linearized by cutting with EcoRI and transformed into B. subtilis 168 strain 1A304 to create strain SH119. Recombinants were selected on nutrient agar plates containing $100 \mu \mathrm{g}$ spectinomycin $\mathrm{ml}^{-1}$ (Steinmetz \& Richter, 1994). The construct was confirmed by Southern blot analysis.

Multiple mutant strains and other constructs were made by transformation with the appropriate chromosomal DNA (see Table 1 for crosses). All molecular biology methods were performed as described by Sambrook et al. (1989).

Swarm plate assay. Swarming motility of strains was measured using nutrient and minimal $0.3 \%$ agar (Difco) plates. Samples $(1 \mu \mathrm{l})$ from overnight $\left(30^{\circ} \mathrm{C}\right)$ liquid cultures were spotted onto swarm plates and incubated at $37^{\circ} \mathrm{C}$ (nutrient agar for $18-22 \mathrm{~h}$, minimal agar for $44-48 \mathrm{~h}$ ) or $25^{\circ} \mathrm{C}$ (nutrient agar for $44-48 \mathrm{~h}$, minimal agar for 68-72 h). Extent of swarming motility was measured as percentage growth diameter relative to the parental control (1A304).

Cell autolysis. Cultures of parental and mutant strains of $B$. subtilis were grown to mid-exponential phase $\left(\mathrm{OD}_{600} 0 \cdot 25-0.3\right)$ in nutrient broth. Lysis of cells, after addition of $0.05 \mathrm{M}$ sodium azide or $5 \mu \mathrm{g}$ cloxacillin $\mathrm{ml}^{-1}(10 \times \mathrm{MIC})$, was followed spectrophotometrically (10 mm path length, Pye Unicam SP6-400 spectrophotometer) while continuing incubation at $37^{\circ} \mathrm{C}$ and 250 r.p.m.

Measurement of cell wall turnover. This was determined in minimal medium using the method of Margot \& Karamata (1992). N-Acetyl-D-[1-14 C]glucosamine was obtained from Amersham and radioactivity of filters and filtrates was estimated in Safefluor-S (LUMAC) scintillation cocktail with a Beckman LS1801 liquid scintillation system.

Preparation of cell-wall-binding protein (CWBP) extracts. Two-litre cultures $\left(\mathrm{OD}_{600} 1.0\right)$ of $B$. subtilis parental and mutant strains were harvested $\left(11000 \mathrm{~g}, 4^{\circ} \mathrm{C}, 10 \mathrm{~min}\right)$, and the cells washed once with ice-cold $50 \mathrm{mM}$ Tris $/ \mathrm{HCl}(\mathrm{pH} 7 \cdot 5)$ and then resuspended in $40 \mathrm{ml}$ of the same buffer. PMSF was 
Table 1. Bacterial strains and plasmids

\begin{tabular}{|c|c|c|}
\hline $\begin{array}{l}\text { Strain or } \\
\text { plasmid }\end{array}$ & Genotype/phenotype & Source/reference* \\
\hline \multicolumn{3}{|c|}{ Strains } \\
\hline \multicolumn{3}{|c|}{ B. subtilis 168} \\
\hline HR & $\operatorname{trpC2}$ & Laboratory stock \\
\hline $1 \mathrm{~A} 304$ & $\operatorname{trp} C 2$ metB5 xin-1 SP $\beta(\mathrm{s})$ & $\mathrm{BGSC} \dagger$ \\
\hline SFG103 & $\operatorname{trp} C 2 w a p A::$ pSFG126 Ery ${ }^{\mathrm{r}}$ & Foster (1993) \\
\hline DP-1 & $\operatorname{trp} C 2 \operatorname{sig} D:: \mathrm{pLM} 5 \mathrm{Cm}^{\mathrm{r}}$ & Helmann et al. (1988) \\
\hline SH125 & $\operatorname{trpC} 2$ lytC::ble & This study \\
\hline SH119 & $\operatorname{trp} C 2$ metB5 xin-1 $S P \beta(\mathrm{s})$ lytD::spc & This study \\
\hline SH115 & $\operatorname{trp} C 2$ metB5 xin-1 SP $\beta(\mathrm{s})$ lytC: : ble & SH125 $\rightarrow 1 \mathrm{~A} 304$ \\
\hline SH118 & $\operatorname{trp} C 2$ metB5 xin-1 SP $\beta(\mathrm{s})$ sigD::pLM5 $\mathrm{Cm}^{\mathrm{r}}$ & $\mathrm{DP}-1 \rightarrow 1 \mathrm{~A} 304$ \\
\hline SH117 & $\operatorname{trp} C 2$ metB5 xin-1 SP $\beta(\mathrm{s}) w a p A:: \mathrm{pSFG} 126 \mathrm{Ery}^{\mathrm{r}}$ & SFG103 $\rightarrow 1 \mathrm{~A} 304$ \\
\hline SH128 & $\operatorname{trpC} 2$ metB5 xin-1 SP $\beta(\mathrm{s})$ lytC: ble lytD::spc & SH119 $\rightarrow$ SH115 \\
\hline SH131 & $\begin{array}{l}\operatorname{trpC} 2 \text { metB5 } x i n-1 S P \beta(\mathrm{s}) \text { lytC:: ble lytD::spc } \\
\operatorname{sig} D:: \text { pLM5 } \mathrm{Cm}^{\mathrm{r}}\end{array}$ & $\mathrm{SH} 118 \rightarrow \mathrm{SH} 128$ \\
\hline SH120 & $\begin{array}{l}\operatorname{trpC} 2 \text { metB5 } x \text { in-1 SP } \beta(\mathrm{s}) \text { wapA::pSFG126 } \mathrm{Ery}^{\mathrm{r}} \\
\text { lytC::ble }\end{array}$ & SH115 $\rightarrow$ SH117 \\
\hline SH121 & $\begin{array}{l}\operatorname{trpC} 2 \text { metB5 } x i n-1 S P \beta(\mathrm{s}) \text { wapA::pSFG126 Ery } \\
\text { lytD::spc }\end{array}$ & SH119 $\rightarrow$ SH117 \\
\hline SH127 & $\begin{array}{l}\operatorname{trp} C 2 \text { metB5 } x i n-1 S P \beta(\mathrm{s}) \text { wapA::pSFG126 } \mathrm{Ery}^{\mathrm{r}} \\
\operatorname{sig} D:: \mathrm{pLM} 5 \mathrm{Cm}^{\mathrm{r}}\end{array}$ & SH118 $\rightarrow$ SH117 \\
\hline SH123 & 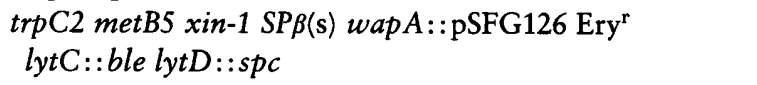 & SH119 $\rightarrow$ SH120 \\
\hline SH124 & 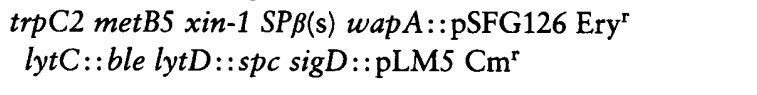 & SH118 $\rightarrow$ SH123 \\
\hline \multicolumn{3}{|l|}{ Plasmids } \\
\hline p6302 & $\begin{array}{l}\text { pJH101 with } 2 \cdot 3 \mathrm{~kb} \text { BamHI-EcoRI insert carrying } B \text {. } \\
\text { subtilis lytC gene }\end{array}$ & Lazarevic et al. (1992) \\
\hline p5102 & $\begin{array}{l}\text { pMTL20EC with } 4 \mathrm{~kb} \mathrm{BamHI} \text { insert carrying } B \text {. subtilis } \\
\text { lytD gene }\end{array}$ & Margot et al. (1994) \\
\hline pTJS43 & $\begin{array}{l}\text { p6302 with } 1.5 \mathrm{~kb} \text { phleomycin resistance cassette }(b l e) \\
\text { inserted into unique } B g l \mathrm{II} \text { site }\end{array}$ & This study \\
\hline pSAB100 & $\begin{array}{l}1.1 \mathrm{~kb} \text { PstI fragment from p5102 cloned into unique PstI } \\
\text { site of pUBS1 }\end{array}$ & This study \\
\hline pSAB101 & $\begin{array}{l}\text { pSAB100 with } 1.2 \mathrm{~kb} \text { spectinomycin resistance cassette } \\
(s p c) \text { inserted into unique } H p a \mathrm{I} \text { site }\end{array}$ & This study \\
\hline
\end{tabular}

* Arrows indicate transformation of recipient strain with donor chromosomal DNA.

† Bacillus Genetic Stock Center, Ohio State University, OH, USA.

added to a concentration of $0.5 \mathrm{mM}$ and the suspension was passed twice through a French pressure cell (Aminco, $180 \mathrm{MPa}, 4^{\circ} \mathrm{C}$ ) to break the cells. Cell walls were sedimented by centrifugation $\left(27000 \mathrm{~g}, 15 \mathrm{~min}, 4^{\circ} \mathrm{C}\right)$ and washed twice by resuspension in ice-cold $50 \mathrm{mM}$ Tris $/ \mathrm{HCl}(\mathrm{pH} 7 \cdot 5)$. The walls were then washed twice with ice-cold $100 \mathrm{mM} \mathrm{NaCl}$ to remove any loosely associated proteins and washed twice again with ice-cold $50 \mathrm{mM}$ Tris $/ \mathrm{HCl}(\mathrm{pH} 7 \cdot 5)$. The cell wall preparations were then stored at $-20^{\circ} \mathrm{C}$. CWBPs were extracted $\left(100^{\circ} \mathrm{C}, 3 \mathrm{~min}\right)$ with SDS sample buffer $[62.5 \mathrm{mM}$ Tris/HCl (pH 6.8), 1 mM EDTA, $1 \%(\mathrm{w} / \mathrm{v})$ SDS, $5 \%(\mathrm{v} / \mathrm{v}) \beta$ mercaptoethanol, $0.0025 \%$ bromophenol blue, $10 \%(\mathrm{v} / \mathrm{v})$ glycerol] and stored at $-20^{\circ} \mathrm{C}$.

SDSPAGE and renaturing SDSPAGE. CWBP samples were analysed by SDS-PAGE (Laemmli, 1970) using $11 \%$ (w/v) acrylamide gels and Coomassie blue staining to visualize proteins. Autolysin activity was detected by renaturing gel electrophoresis as described by Foster (1992) using purified $B$. subtilis vegetative cell walls as the substrate.

Cell separation. To study filamentation of parental and mutant strains using phase-contrast microscopy, cells were harvested at specific points during growth in nutrient broth and fixed in ethanol using the method of Hauser \& Errington (1995). Cells were mounted on Poly-Prep poly-L-lysine-coated slides (Sigma) and stained with methylene blue. To study macrofilamentation (boli), cultures $(10 \mathrm{ml})$ of $B$. subtilis strains were grown for $18 \mathrm{~h}$ at $25^{\circ} \mathrm{C}$ with gentle shaking (45 r.p.m.) and carefully poured into Petri dishes for photography. For electron microscopy, samples of late-exponential or early-stationary phase cultures of $B$. subtilis parental and mutant strains were taken.

$\mathbf{N}$-terminal sequence determination. A CWBP sample of the multiple mutant strain ( $\mathrm{SH} 124$, wapA lytC lytD sigD) was 
(a)
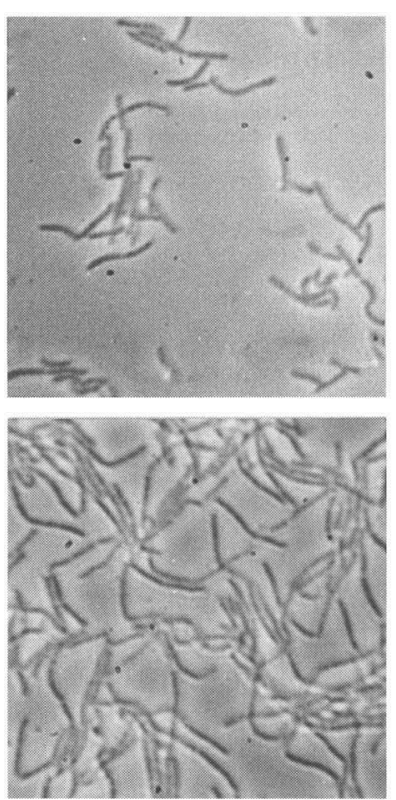

SH118
SH115
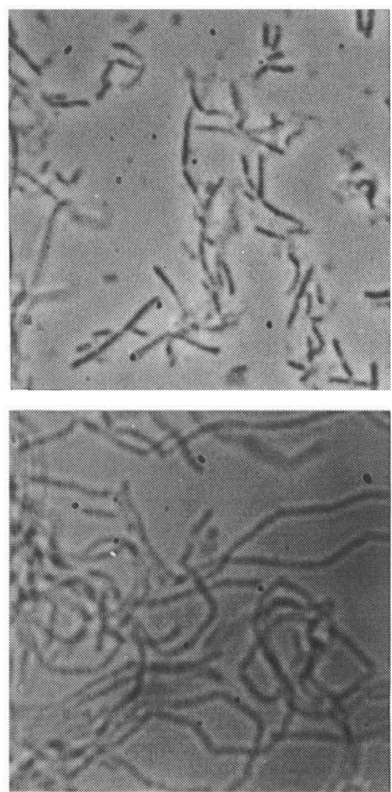

SH128
SH119
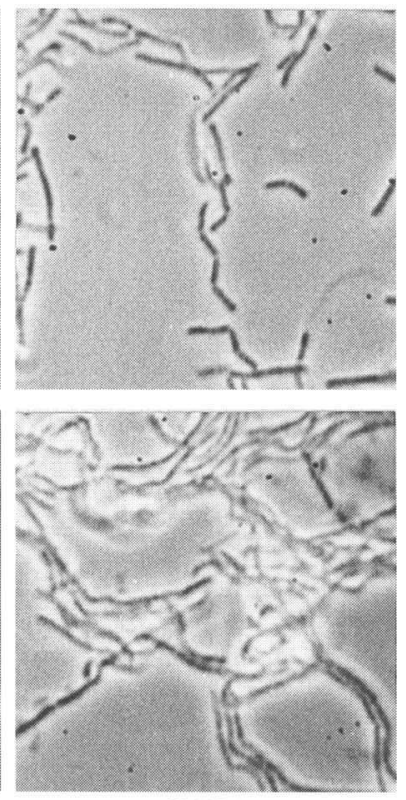

SH131

(b) $1 \mathrm{~A} 304$

SH115

SH119

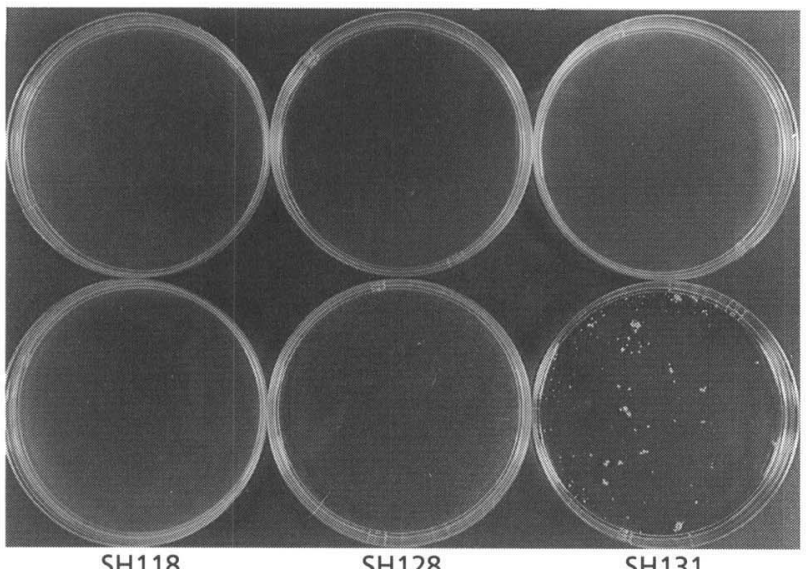

SH128

SH131

(c)

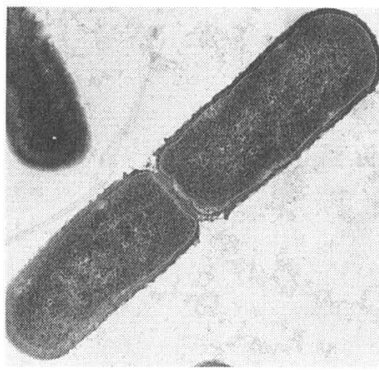

1A304

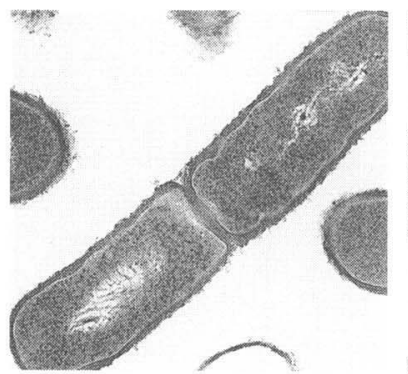

SH115

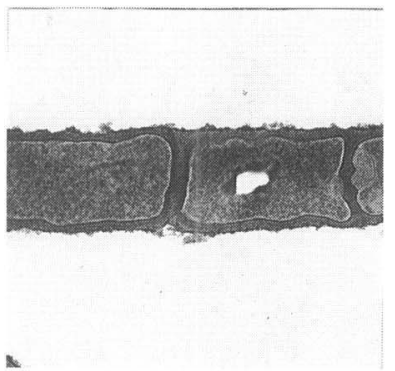

SH131

Fig. 1. Cell separation of stationary-phase cultures of $B$. subtilis strains illustrated by phase-contrast micrographs (bar, $10 \mu \mathrm{m})(a)$, liquid cultures (b) and electron micrographs (bar, $1 \mu \mathrm{m})(\mathrm{c})$. Phase-contrast samples were stained and mounted as described in Methods. Liquid cultures (10 ml nutrient broth) were grown for $18 \mathrm{~h}$ at $25^{\circ} \mathrm{C}$ with gentle shaking (45 r.p.m.) and carefully poured into Petri dishes for photography. Electron micrograph samples were fixed in glutaraldehyde, embedded in araldite and sections stained in uranyl acetate followed by Reynolds lead citrate solution. Photographs are of representative samples from at least two independently grown cultures. 
subjected to $11 \%$ SDS-PAGE and then electrophoretically transferred onto ProBlott membrane (Applied Biosystems) as described by the manufacturer. A number of bands, corresponding to lytic bands on the renaturing gel, were excised and the peptide sequence was determined with an Applied Biosystems $476 \mathrm{~A}$ protein sequencer.

\section{RESULTS}

\section{Construction of multiple lytic enzyme mutants of $B$. subtilis 168}

B. subtilis 168 strain $1 \mathrm{~A} 304$ was used as the parental strain for mutant construction as it is unable to express three phage lytic enzymes usually associated with $B$. subtilis 168 . The lytC gene was disrupted by insertional inactivation using a phleomycin resistance cassette. The lyt $D$ gene was similarly inactivated using a spectinomycin resistance cassette. The correct insertion in both genes was confirmed by Southern blotting and renaturing gel analysis (results not shown). A set of isogenic strains was constructed (Table 1) to allow the individual and combined roles of the autolysins and associated components to be assessed.

\section{Cell growth and division}

All strains grew at equivalent rates in nutrient broth and reached the same final $O_{600}$ value (results not shown). However, accurate $\mathrm{OD}_{600}$ readings could not be obtained for strain SH131 (lytC lytD sigD) for reasons that are explained below. The effects of the single or multiple mutations on cell morphology during growth in batch culture were studied by phase-contrast microscopy. Strains HR and 1A304 showed identical morphology and division kinetics (results not shown). The chains of cells of the parental strain were shortest at the beginning of growth (lag phase), gradually becoming longer through exponential growth, reaching their maximum length (four to eight cells) as the cultures entered stationary phase. During stationary phase, the chains gradually shortened, eventually becoming unicellular and motile. All strains showed similar cellular dimensions and pattern of filamentation during growth (the degree of filamentation depending on the strain examined). Fig. 1 shows phase-contrast micrographs, liquid cultures and electron micrographs of representative samples from at least two cultures of selected strains. Strains SH115 (lytC) and SH119 (lytD) showed only very slight increases in chain length relative to the parental strain (Fig. 1a), four to ten cells per chain compared with two to six. A strain inactivated in $\operatorname{sig} D$ (SH118) was somewhat filamentous (12-18 cells per chain) (Fig. 1a). When $l y t C$ and $l y t D$ mutations were combined (SH128), extremely long chains of cells were formed that spanned many fields of view and often wrapped around each other forming large lumps when viewed by phase-contrast microscopy (Fig. 1a). These results suggested that both $\mathrm{LytC}$ and $\mathrm{LytD}$ had a role in cell separation but that they could mutually compensate for the lack of each other. The lytC lytD sigD triple mutant (SH131) had a similar phenotype to SH128 (lytC lytD) when viewed under the phase-contrast microscope
(Fig. 1a), but in liquid culture large boli up to $10 \mathrm{~mm}$ across were formed (Fig. 1b). This suggested that another SigD-controlled enzyme (or enzymes) is involved in cell separation, as it was only in the multiple mutant strain that boli were formed. When the strains were viewed by electron microscopy it was noted that in the lytC-inactivated strain (SH115), the thickness of the cell walls $(45-60 \mathrm{~nm})$ and septa $(60-95 \mathrm{~nm})$ was greater than in wild-type (1A304) walls $(25-40 \mathrm{~nm})$ and septa $(45-70 \mathrm{~nm})$ (Fig. 1c). Also the walls were rougher when compared to the wild-type (Fig. 1c). The walls and septa of SH131 (lytC lytD sigD) showed even greater thickness at $60-70 \mathrm{~nm}$ and $100-120 \mathrm{~nm}$, respectively (Fig. 1c).

\section{Swarming motility}

All strains bearing mutations in specific autolysin genes were motile in stationary phase when viewed by phasecontrast microscopy, apart from strain $\mathrm{SH} 128$ (lytC lytD), the immotility of which may be due to its very high degree of filamentation. The strain inactivated in sigD alone ( $\mathrm{SH} 118)$ was immotile as this sigma factor controls expression of genes essential for flagellar motility (Helmann et al., 1988). The strains were also analysed by growth on swarm plates since these are a measure not only of bacterial motility but also of chemotaxis. On swarm plates motile strains form a halo of diffuse growth around the tightly packed central colony. Swarming motility was measured on nutrient and minimal plates at 37 and $25^{\circ} \mathrm{C}$ and the results were expressed as the percentage colony diameter relative to the parental strain (Table 2). Strain SH115 (lytC) showed a reduction in swarming motility, the halo diameter varying between 43 and $67 \%$ that of the parental strain, depending on the medium used and the temperature of incubation. Strain SH119 (lytD) also showed a reduction in motility. It swarmed to 40 and $34 \%$ the diameter of the parental strain on nutrient plates, and 77 and $60 \%$ the diameter of the parental strain on minimal plates at 37 and $25^{\circ} \mathrm{C}$, respectively. Strain SH118 (sigD) does not express the major flagellar protein, flagellin, and so it was predicted to be nonmotile. However, it did apparently swarm to an extent, forming a small halo on nutrient plates $(20$ and $16 \%$ at 37 and $25^{\circ} \mathrm{C}$, respectively) and producing quite a large halo on minimal plates (48 and $52 \%$ at 37 and $25^{\circ} \mathrm{C}$, respectively). Strain SH128 (lytC lytD) does not swarm at all, forming no halo whatsoever under any conditions $(11-16 \%)$. These results suggest that LytD and, to a lesser extent, LytC have a role in swarming motility and can partially compensate for each other.

\section{Cell autolysis}

Cellular lysis of $B$. subtilis was measured by adding either sodium azide or cloxacillin to exponentially growing cells and following lysis spectrophotometrically. Fig. 2(a) shows representative data from three independent experiments following lysis of cultures after addition of sodium azide. Lysis of 1A304 began immediately after the addition of sodium azide 
Table 2. Swarm plate assay of $B$. subtilis strains

\begin{tabular}{|c|c|c|c|c|c|}
\hline \multirow[t]{2}{*}{ Strain } & \multirow[t]{2}{*}{ Genotype } & \multicolumn{4}{|c|}{ Swarm diameter $(\%)^{*}$} \\
\hline & & $\mathrm{NA}, 37^{\circ} \mathrm{C}$ & $\mathrm{NA}, 25^{\circ} \mathrm{C}$ & $\mathrm{MA}, 37^{\circ} \mathrm{C}$ & $\mathrm{MA}, 25^{\circ} \mathrm{C}$ \\
\hline SH115 & $\operatorname{lyt} \mathrm{C}^{a}$ & $65 \pm 5 \cdot 5$ & $43 \pm 2 \cdot 3$ & $48 \pm 2 \cdot 9$ & $67 \pm 1 \cdot 4$ \\
\hline SH119 & $\operatorname{lyt} \mathrm{D}^{a}$ & $40 \pm 1 \cdot 7$ & $34 \pm 3 \cdot 3$ & $77 \pm 6 \cdot 5$ & $60 \pm 2 \cdot 3$ \\
\hline SH118 & $\operatorname{sig} D$ & $20 \pm 1 \cdot 1^{b}$ & $16 \pm 1 \cdot 8^{b}$ & $48 \pm 9 \cdot 4^{a}$ & $52 \pm 3 \cdot 3^{a}$ \\
\hline SH128 & lytC lyt $D^{b}$ & $16 \pm 1 \cdot 5$ & $15 \pm 1 \cdot 0$ & $13 \pm 0 \cdot 3$ & $11 \pm 0 \cdot 7$ \\
\hline
\end{tabular}

* Swarm diameters were measured on nutrient agar (NA) or minimal agar (MA) plates at 37 or $25^{\circ} \mathrm{C}$ as described in Methods. Results are expressed as a percentage of the parental strain (1A304) (70$85 \mathrm{~mm}) \pm$ SEM. SEM equals $\sigma_{n-1} / \sqrt{ } n$ (Rashid et al., 1995), where $n=14(a)$ or $5(b)$.

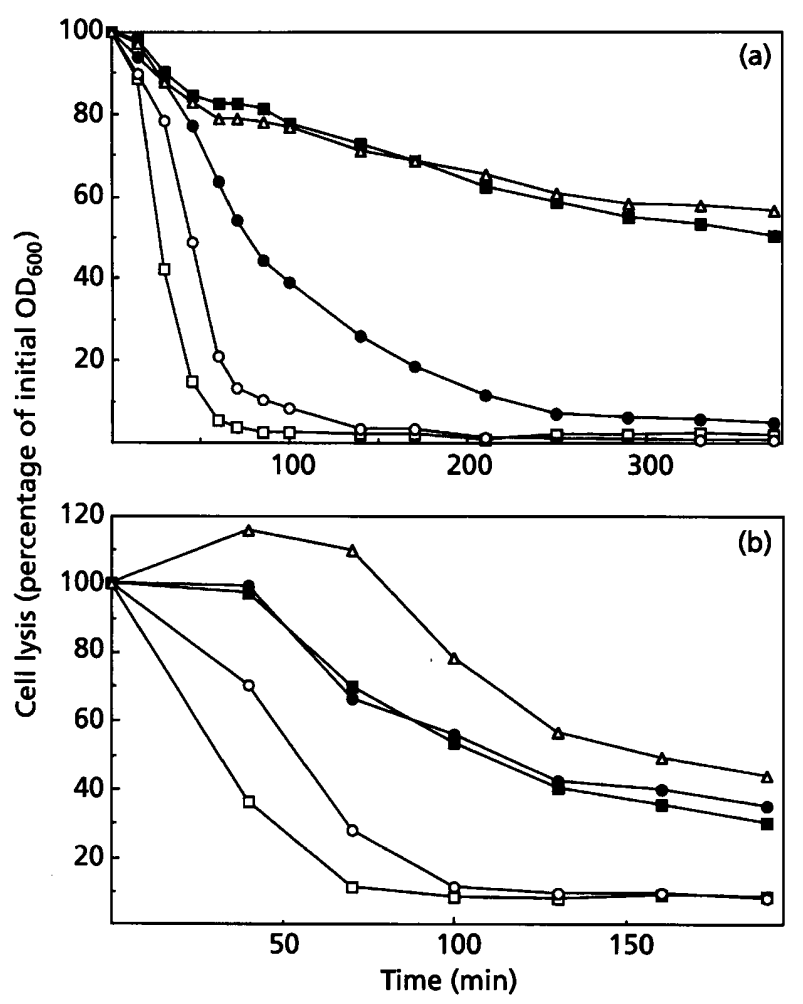

Fig. 2. Cell lysis of exponential-phase cultures of B. subtilis wild-type and mutant strains after the addition of sodium azide (a) or cloxacillin (b). Sodium azide (0.05 M) or cloxacillin $(5 \mu \mathrm{g}$ $\left.\mathrm{ml}^{-1}, 10 \times \mathrm{MIC}\right)$ was added to exponentially growing cultures $\left(O D_{600} 0.25-0.3\right)$ and lysis followed spectrophotometrically at $600 \mathrm{~nm} . \square, 1 \mathrm{A304}$ (parent); 0, SH115 (lytC); O, SH119 (lytD); - SH118 (sigD); $\triangle$, SH128 (lytC lytD). Data are representative of three independent experiments.

and $>95 \%$ lysis had occurred within $60 \mathrm{~min}$. Strain SH115 $(l y t C)$ showed a marked reduction in rate of lysis, with only 17 and $49 \%$ reduction in $\mathrm{OD}_{600}$ occurring after 60 and $370 \mathrm{~min}$, respectively. Conversely, strain SH119 (lytD) was only very slightly reduced in the rate of lysis, and this may not be significant. In fact strain SH128 (lytC lytD) lysed at the same rate as strain SH115 (lytC), suggesting that LytD is not involved in sodiumazide-induced cellular lysis. Strain SH118 (sigD) also showed a reduction in the rate of lysis compared to parental $1 \mathrm{~A} 304$ with 36 and $95 \%$ lysis after 60 and $370 \mathrm{~min}$, respectively. The decreased rate of lysis in SH118 was probably due to the reduced level of LytC or other SigD-controlled components. This phenomenon could not be investigated further as strain SH131 (lytC $l y t D \operatorname{sig} D$ ) was too filamentous to obtain accurate $\mathrm{OD}_{600}$ readings.

Lysis of $B$. subtilis after the addition of the cell-wallsynthesis-inhibiting antibiotic cloxacillin was studied with three independent experiments and representative data are shown in Fig. 2(b). The parental strain (1A304) lost $>90 \% \mathrm{OD}_{600}$ after $70 \mathrm{~min}$. Both SH115 $(l y t C)$ and SH119 (lytD) had a reduced rate of lysis, losing 30 and $72 \%$, respectively, of initial $\mathrm{OD}_{600}$ after $70 \mathrm{~min}$. Strain SH118 (sigD) lysed at the same rate as SH115 (lytC), but in SH128 (lytC lytD) lysis was delayed by $40 \mathrm{~min}$ and then proceeded slowly. Thus both LytC and LytD have a role in antibiotic-induced lysis and are partially able to compensate for each other in this process.

\section{Cell wall turnover}

As $B$. subtilis cells grow and divide, old cell wall material is shed at the outer surface, presumably by the action of endogenous autolysins. This cell wall turnover was analysed in exponentially growing cells by two independent pulse-chase experiments using $N$-acetyl-D$\left[1-{ }^{14} \mathrm{C}\right]$ glucosamine to label the cell wall peptidoglycan. Representative results are shown in Fig. 3. Strain 1A304 (parental) lost $>90 \%$ of incorporated labelled material after $90 \mathrm{~min}$, which corresponds to about three generations. Strain SH115 (lytC) showed a marked reduction in the rate of wall turnover, with only $37 \%$ of incorporated label lost after $90 \mathrm{~min}$. There was no delay in the initiation of release of labelled material. Strain SH119 (lytD) had almost identical wall turnover kinetics when compared with the parental strain. However, strain SH128 (lytC lytD) showed a greater reduction in wall turnover than SH115 (lytC). Thus both LytC and LytD were shown to have a role in wall turnover. The absence of LytD could, however, be totally compensated for by LytC, but LytD could only partially compensate for the lack of LytC. Strain SH118 (sigD) had a reduced rate of wall turnover when compared to the parental strain $(56 \%$ release of label after $90 \mathrm{~min}$ ) but the rate of 


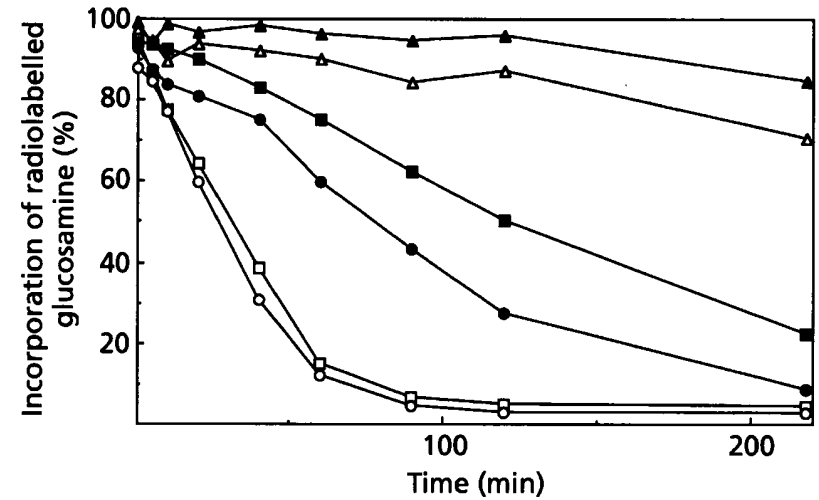

Fig. 3. Cell wall turnover of exponential-phase cultures of $B$. subtilis wild-type and mutant strains. Cells were labelled for five generations with $\mathrm{N}$-acetyl-D-[1-14C]glucosamine and transferred to chase medium (Margot \& Karamata, 1992). Samples, withdrawn at regular intervals, were precipitated with ice-cold 5\% trichloroacetic acid, filtered and washed. Radioactivity of filters and filtrates was determined and the proportion of label released through wall turnover was calculated as: $100 \times$ d.p.m. on filters/(d.p.m. on filters + d.p.m. in filtrates). $\square, 1$ A304 (parent); $\square$, SH115 (lytC); O, SH119 (lytD); . SH118 (sigD); $\triangle$, SH128 (lytC lytD); $\triangle$, SH131 (lytC lytD sigD). Data are representative of two independent experiments.

wall turnover was still greater than that of strain $\mathrm{SH} 115$ $(l y t C)$. This could be explained by the small proportion of lytC expression remaining in SH118 (sigD). Strain SH131 (lytC lytD sigD) exhibited only $5 \%$ wall turnover after 90 min, even less than SH128 (lytC lytD), which exhibited $15 \%$ wall turnover after 90 min. This suggested that a further, as yet unknown, SigD-controlled autolysin has a role in cell wall turnover.

\section{Differentiation}

No observable, significant differences were seen in sporulation frequency and germination rate between any of the strains studied in this work.

\section{Analysis of minor vegetative cell autolysins}

CWBP extracts from purified native cell walls give a fraction enriched for autolysins, as autolysins are primarily wall-associated. The wapA gene encodes the proform of three major CWBPs in B. subtilis (Foster, 1993). Strains were constructed bearing the wapA mutation as this reduced total CWBP levels, allowing easier analysis and study of minor autolysins. Inactivation of wapA had no effect on the autolysin profile (Fig. 4, lane 2), cell morphology or pattern of cell division (results not shown). Renaturing SDS-PAGE analysis of CWBP preparations from the mutant strains confirmed the absence of LytC and LytD in the relevant strains (Fig. 4, lanes 3 and 4). Otherwise masked by the presence of LytC was another lytic enzyme of $49 \mathrm{kDa}$ (lanes 3 and 6) (Smith et al., 1996), which may correspond to the CWBP49' of Margot \& Karamata (1992). Ex-

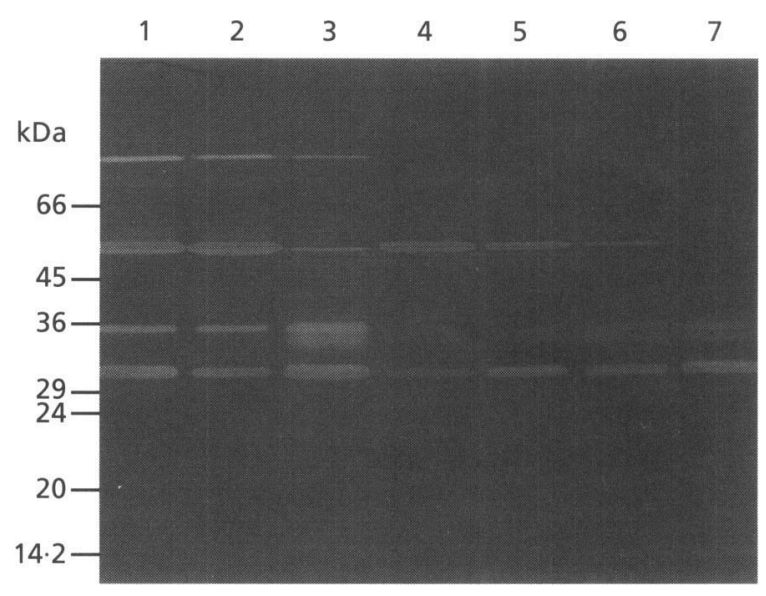

Fig. 4. Renaturing SDS-PAGE of CWBP extracts of B. subtilis 168 wild-type and mutant strains. Samples were prepared as described in Methods and renaturing SDS-PAGE was carried out as previously described (Foster, 1992) using purified B. subtilis cell walls as the substrate. Lanes: 1, 1 A304 (parent); 2, SH117 (wapA); 3, SH120 (wapA lytC); 4, SH121 (wapA lytD); 5, SH118 (wapA sigD); 6, SH123 (wapA lytC lytD); 7, SH124 (wapA lytC lytD sigD).

pression of this enzyme is controlled by SigD as it was missing in the $\operatorname{sig} D$ background of the multiple mutant strain (SH124, lane 7). Despite the inactivation of both major vegetative autolysins and $\operatorname{sig} D$, there were still at least ten bands of autolytic activity in the multiple mutant strain (lane 7). We have now obtained $\mathrm{N}$ terminal sequences of seven of the corresponding proteins (results not shown). It is interesting to note that many of these are distinct from each other and so may correspond to different gene products or proteolytic products of larger proforms.

\section{DISCUSSION}

A set of isogenic strains has been created which has allowed in-depth physiological analysis of the roles of the major autolysins of $B$. subtilis. The parental strain used in these studies was $1 \mathrm{~A} 304$, which is unable to express prophage lytic enzymes. This removes contaminating phage lytic activity. In all pertinent respects 1A304 is identical to HR (B. subtilis $168 \operatorname{trpC2}$ ) which can express phage enzymes. Mutants of $B$. subtilis 168 inactivated in the two major vegetative autolysin genes (lytC and $l y t D)$ have previously been constructed and studied phenotypically (Margot \& Karamata, 1992; Margot et al., 1994; Rashid et al., 1993, 1995). However, our study has revealed a number of phenotypes that have not previously been recorded and has shown hitherto unknown roles for the autolysins during vegetative growth.

Margot et al. (1994) noted that a strain inactivated in lyt $\mathrm{C}$ showed a $60 \mathrm{~min}$ delay in the release of radiolabelled cell-wall material, which subsequently occurred at a rate identical to that of the wild-type strain. Also, the lytD mutation had no effect on wall turnover either singly or in combination with lytC. However, our 
experiments (Fig. 3) showed that SH115 (lytC) had a reduced rate of wall turnover but with no delay. Strain SH119 (lytD) was unaffected in wall turnover, but strain SH128 (lytC lytD) was reduced in the rate of wall turnover to a greater extent than SH115 (lytC). This shows that both $\mathrm{LytC}$ and $\mathrm{LytD}$ have a role in cell wall turnover. The absence of LytD could be totally compensated for by LytC, but LytD could only partially compensate for the lack of LytC. A strain inactivated in $\operatorname{sig} D$, the gene that encodes $\sigma^{\mathrm{D}}$, the sigma factor controlling motility and chemotaxis (Helmann et al., 1988), was slightly reduced in the rate of wall turnover. The expression of both $l y t C$ and $l y t D$ is mostly under the control of SigD and so this reduced rate of turnover may be due to the lower levels of these enzymes in this strain. However, the greater reduction in the rate of wall turnover of strain SH131 (lytC lytD sigD) compared to SH128 (lytC lytD) suggests that a further SigD-controlled autolysin(s) may be involved.

It has long been suggested that one of the roles for autolysins in bacteria is to facilitate separation of cells after septation (Lominski et al., 1958; Forsberg \& Rogers, 1971). Although filamentous mutants of $B$. subtilis have been isolated (Fein \& Rogers, 1976), these mutations were subsequently found to be located in regulatory genes (Pooley \& Karamata, 1984). These have been characterized as sigD (Helmann et al., 1988) and $\sin$ (Gaur et al., 1986), both of which show reduced autolysin levels. It was postulated that the reason for the filamentation of $\operatorname{sig} D$ and $\sin$ strains was the decreased amounts of LytC and LytD (Fein \& Rogers, 1976). Surprisingly, when the structural genes encoding LytC and LytD were inactivated this had no effect on cell separation (Margot et al., 1994; Rashid et al., 1995). However, our mutants show notable filamentation changes compared to the parental strain (Fig. 1a). More physiological growth conditions, for example lower temperature, may affect the degree of filamentation. Also, to reduce the physical disruption effects on chain length, cultures were shaken at lower speeds. Aeration was maintained by use of a low culture-to-flask volume ratio $(10 \mathrm{ml}$ nutrient broth in a $250 \mathrm{ml}$ flask). By growing our mutants under these conditions we observe substantial increases in chain lengths which highlights further differences between the strains (Fig. 1b). All strains showed similar kinetic patterns of filamentation to each other throughout growth. Single inactivations of $l y t C$ and $l y t D$ showed slight increases in cell chain lengths compared to the parental strain. The very obvious filamentous phenotype of SH128 (lytC lytD) suggests that both of the major vegetative enzymes have roles in cell separation. These enzymes can compensate for the lack of each other, as it is only when both genes are inactivated that there is gross filamentation. As described previously (Helmann et al., 1988), a sigDinactivated strain is somewhat filamentous, suggesting that the autolysins involved in septal peptidoglycan hydrolysis are under the control of $\sigma^{\mathrm{D}}$. The lytC gene is $70-90 \%$ and $l y t D$ is $95 \%$ under the control of SigD (Lazarevic et al., 1992; Kuroda \& Sekiguchi, 1993;
Margot et al., 1994; Rashid et al., 1995). Therefore, the filamentation in a sigD strain may just be due to the reduced levels of LytC and LytD in this mutant. However, seemingly at least one other SigD-controlled enzyme is involved in cell separation, as only strain SH131 (lytC lytD sigD) forms large filamentation masses, or boli, in liquid culture (Fig. 1b). A candidate for the third cell separation enzyme under the control of $\mathrm{SigD}$ is the $49 \mathrm{kDa}$ autolysin identified by renaturing gel electrophoresis in strain SH123 (Fig. 4, lane 6) (Smith et al., 1996). This is probably the protein described as CWBP49' by Margot \& Karamata (1992), as this is also SigD-controlled. Therefore, at least three enzymes are involved in cell separation, all with mutually compensatory roles. This functional redundancy suggests that the enzymes are performing similar functions in cell separation.

The minor $49 \mathrm{kDa}$ enzyme may also be involved in cell wall turnover as SH131 (lytC lytD sigD) shows reduced turnover compared to SH128 (lytC lytD). Thus LytC, LytD and the minor $49 \mathrm{kDa}$ enzyme are also implicated in cell wall turnover, and so it is possible that cell separation is simply a function of cell wall turnover. Wall material is constantly being cleaved and released and when enough of this activity has occurred at a nascent septum the cells can separate. This hypothesis is supported by electron microscopy, as both SH115 (lytC) and SH131 (lytC lytD sigD) have thickened septa (Fig. 1c), concomitant with reduced wall turnover. However, strain SH118 (sigD) is more filamentous than strain SH115 (lytC) but is less affected in wall turnover. Furthermore, it has been shown that wall turnover is slower at the poles compared to the cylindrical regions of the cell (Archibald \& Coapes, 1976), although the poles do still turn over (Clarke-Sturman et al., 1989). Although cell wall turnover may have a role to play in cell separation, there is seemingly a more specialized process occurring. What is special about the poles? It could be that autolysins are preferentially located here. This seems unlikely, however, as both LytC and LytD are involved in cell wall turnover which occurs primarily over the cylindrical surface. A change in the substrate peptidoglycan would allow autolysins to fulfil roles in cell wall turnover and cell separation. How the activity of autolysins is controlled at the post-translational level is unknown, but it has been postulated that it is due to membrane energization (Jolliffe et al., 1981), lipoteichoic acid inhibition (Fischer et al., 1981), substrate conformation (Koch et al., 1985), the wall ionic environment (Cheung \& Freese, 1985) and extracellular protease activity (Jolliffe et al., 1980). Control may, therefore, be mediated by electrochemical interactions directly or indirectly by affecting the three-dimensional conformation of the substrate. Peptidoglycan conformation at the outer surface of the cell may be equivalent to that of the central divide of the nascent septum, allowing any one of a number of enzymes to act at either of these locations. In this model, when the cell is killed by sodium azide, destroying the proton motive force across the membrane, the conformation of the cell wall 
peptidoglycan is likely to change, allowing unrestricted autolysin activity and generalized cell lysis. There are, however, further levels of control, as LytD cannot compensate for the lack of LytC in this process. Antibiotic-induced death and lysis is a more complex process and its mechanism has yet to be fully explained (Rogers et al., 1983).

Fein (1979) noted that mutants FJ3, FJ6, FJ7 and Nil5 are $90 \%$ deficient in both the amidase (LytC) and the glucosaminidase $(\mathrm{LytD})$ and are non-motile, suggesting that autolysins are required for flagellar morphogenesis. These mutations were found to be in regulatory genes (Pooley \& Karamata, 1984) sigD and sin (Helmann et al., 1988; Gaur et al., 1986), which control the expression of flagellar proteins. Previous results are conflicting as to the role of the major autolysins in swarming. Margot et al. (1994) reported that a strain inactivated in $l y t C$ alone showed a reduction in motility on swarm plates, but a lytD single mutant was unaffected. Rashid et al. (1993) reported that a lytC lytD doubly inactivated strain showed a marked reduction in swarming motility, but the single mutants were unaffected. However, more recently published work has shown that both the $l y t C$ and $l y t D$ single mutants were reduced in swarming motility (Rashid et al., 1995). In our results, strains SH115 (lytC) and SH119 (lytD) were reduced in swarming motility, SH119 more so than SH115. Strain SH128 (lytC lytD) was non-motile on swarm plates (Table 2). When sigD, the chemotaxis and motility sigma factor gene, is inactivated the strain is somewhat filamentous. Autolysins involved in hydrolysis of septal peptidoglycan, including LytC and $\mathrm{LytD}$, are presumably co-regulated with the motility genes because it is advantageous for the bacteria to split into single cells when they become motile, to allow them to move more freely and disperse. CWBP49', which we postulate has a role in cell separation and cell wall turnover, is under the control of SigD and so it can be predicted that inactivation of the gene for this enzyme, either singly or in concert with other autolysin genes, would also impair motility. The lack of motility of SH128 (lytC lytD) is probably due to the fact that this strain is highly filamentous and so cannot move over the surface. The reduced swarming of strains SH115 (lytC) and SH119 $(l y t D)$ is probably due to the slightly more filamentous phenotype of these mutants. Both of these strains are motile when viewed microscopically. However, even two unseparated cells will be unable to chemotax effectively, as they will be transducing signals independently and will be attempting to roll and tumble in different directions from each other. Two unseparated cells will, therefore, form a type of pushmi-pullyu (a llama with a head at both ends in 'The Story of $\mathrm{Dr}$ Dolittle' by Hugh Lofting). Therefore, the reduction in motility on swarm plates may be a result of their filamentous phenotypes. However, despite the obvious difference in motility between the $l y t C$ and $l y t D$ strains, the difference in cell separation of these strains is not great. This leaves open the possibility that autolysins are involved in other swarming-associated functions. How flagellum extrusion through the cell wall occurs is unknown but it may well involve the action of autolysins (Dijkstra \& Keck, 1996). The apparent motility observed in the sigD strain SH118 (Table 2) may be due to colony spreading as a function of growth. In support of this, the more filamentous strain (SH128) swarms less.

Only two vegetatively expressed autolysins in B. subtilis (LytC and LytD) have been studied at the molecular level. In this study we have identified roles for these enzymes in cell separation, motility, cell lysis and cell wall turnover. We have proposed that a minor $49 \mathrm{kDa}$ autolysin, controlled by SigD, has roles in cell separation and cell wall turnover. To determine the predicted roles of this enzyme it will be necessary to inactivate its structural gene. As this protein is produced at very low levels it has so far proved impracticable to purify enough protein for analysis. To circumvent this problem we have constructed a strain bearing an inducible sigD gene in a flgM (anti-sigma factor) (Fredrick \& Helmann, 1996) background. Overexpression of the $49 \mathrm{kDa}$ enzyme in this strain will allow further analysis.

There are many minor autolysins associated with vegetative cells of $B$. subtilis (Fig. 4, lane 7). Also, the $B$. subtilis genome sequencing project has revealed several autolysin homologues. Thus there are many more autolysins still to be analysed, whose function and regulation are completely unknown. We propose that the only rational way to investigate the complex interactive roles of these enzymes is by the study of isogenic multiple mutants using a combinatorial approach. We have set the foundations for this longer term strategy and have already revealed unexpected roles for the major enzymes. The central question still remains as to why this organism, and many others, have so many autolytic enzymes with seemingly overlapping functions.

\section{ACKNOWLEDGEMENTS}

This work was supported by the BBSRC and the Royal Society. We are grateful to M. Chamberlin, G. Murphy and D. Karamata for gifts of strains and plasmids.

\section{REFERENCES}

Anagnostopoulos, C. \& Spizizen, J. (1961). Requirements for transformation in Bacillus subtilis. J Bacteriol 81, 741-746.

Archibald, A. R. \& Coapes, H. E. (1976). Bacteriophage SP50 as a marker for cell wall growth in Bacillus subtilis. J Bacteriol 125, 1195-1206.

Cheung, H. Y. \& Freese, E. (1985). Mono-valent cations enable cell-wall turnover of the turnover-deficient lyt-15 mutant of Bacillus subtilis. J Bacteriol 161, 1222-1225.

Clarke-Sturman, A. J., Archibald, A. R., Hancock, I. C., Harwood, C. R., Merad, T. \& Hobot, J. A. (1989). Cell wall assembly in Bacillus subtilis: partial conservation of polar wall material and the effect of growth conditions on the pattern of incorporation of new material at the polar caps. J Gen Microbiol 135, 657-665.

Cutting, S. M. \& Vander Horn, P. B. (1990). Genetic analysis. In Molecular Biology Methods for Bacillus, pp. 27-74. Edited by C. R. Harwood \& S. M. Cutting. Chichester: John Wiley. 
Dijkstra, A. J. \& Keck, W. (1996). Peptidoglycan as a barrier to transenvelope transport. J Bacteriol 178, 5555-5562.

Fein, J. E. (1979). Possible involvement of bacterial autolysin enzymes in flagellar morphogenesis. J Bacteriol 137, 933-946.

Fein, J.E. \& Rogers, H. J. (1976). Autolytic enzyme deficient mutants of Bacillus subtilis 168. J Bacteriol 127, 1427-1442.

Fischer, W., Rosel, P. \& Koch, H. U. (1981). Effect of alanine ester substitution and other structural features of lipoteichoic acids on their inhibitory activity against autolysins of Staphylococcus aureus. J Bacteriol 146, 467-475.

Forsberg, C. \& Rogers, H. J. (1971). Autolytic enzymes in growth of bacteria. Nature 229, 272-273.

Foster, S. J. (1991). Cloning, expression, sequence analysis and biochemical characterization of an autolytic amidase of Bacillus subtilis 168 trpC2. J Gen Microbiol 137, 1987-1998.

Foster, S. J. (1992). Analysis of the autolysins of Bacillus subtilis 168 during vegetative growth and differentiation by using renaturing polyacrylamide gel electrophoresis. J Bacteriol 174 , $464-470$.

Foster, S. J. (1993). Molecular analysis of three major wallassociated proteins of Bacillus subtilis 168: evidence for processing of the product of a gene encoding a $258 \mathrm{kDa}$ precursor two-domain ligand-binding protein. Mol Microbiol 8, 299-310.

Fredrick, K. \& Helmann, J. D. (1996). FlgM is a primary regulator of $\sigma^{\mathrm{D}}$ activity, and its absence restores motility to a $\sin R$ mutant. J Bacteriol 178, 7010-7013.

Gaur, N. K., Dubnau, E. \& Smith, I. (1986). Characterization of a cloned Bacillus subtilis gene that inhibits sporulation in multiple copies. J Bacteriol 168, 860-869.

Ghuysen, J.-M., Tipper, D. J. \& Strominger, J. L. (1966). Enzymes that degrade bacterial cell walls. Methods Enzymol 8, 685-699.

Hauser, P. M. \& Errington, J. (1995). Characterization of cell cycle events during the onset of sporulation in Bacillus subtilis. J Bacteriol 177, 3923-3931.

Helmann, J. D., Màrquez, L. M. \& Chamberlin, M. J. (1988). Cloning, sequencing, and disruption of the Bacillus subtilis $\sigma^{28}$ gene. J Bacteriol 170, 1568-1574.

Herbold, D. R. \& Glaser, L. (1975). Bacillus subtilis N-acetylmuramic acid L-alanine amidase. J Biol Chem 250, 1676-1682.

Jolliffe, L. K., Doyle, R. J. \& Streips, U. N. (1980). Extracellular proteases modify cell wall turnover in Bacillus subtilis. J Bacteriol 141, 1199-1208.

Jolliffe, L. K., Doyle, R. J. \& Streips, U. N. (1981). The energised membrane and cellular autolysis in Bacillus subtilis. Cell 25, 753-763.

Koch, A. L., Kirchner, G., Doyle, R. J. \& Streips, U. N. (1995). How does a Bacillus split its septum right down the middle? Ann Inst Pasteur Microbiol 136, 91-98.

Kuroda, A. \& Sekiguchi, J. (1991). Molecular cloning and sequencing of a major Bacillus subtilis autolysin gene. J Bacteriol 173, 7304-7312.

Kuroda, A. \& Sekiguchi, J. (1993). High-level transcription of the major Bacillus subtilis autolysin operon depends on expression of the sigma $\mathrm{D}$ gene and is affected by a $\sin (f l a D)$ mutation. $J$ Bacteriol 175, 795-801.

Kuroda, A., Rashid, M. H. \& Sekiguchi, J. (1992). Molecular cloning and sequencing of the upstream region of the major Bacillus subtilis autolysin gene: a modifier protein exhibiting sequence homology to the major autolysin and the spoIID product. J Gen Microbiol 138, 1067-1076.

Laemmli, U. K. (1970). Cleavage of structural proteins during the assembly of the head of bacteriophage T4. Nature 227, 680-685.

Lazarevic, V., Margot, P., Soldo, B. \& Karamata, D. (1992) Sequencing and analysis of the Bacillus subtilis lytRABC divergon: a regulatory unit encompassing the structural genes of the $\mathrm{N}$-acetylmuramoyl-L-alanine amidase and its modifier. $J$ Gen Microbiol 138, 1949-1961.

Lominski, I., Cameron, J. \& Wyllie, G. (1958). Chaining and unchaining of Streptococcus faecalis: a hypothesis of the mechanism of bacterial separation. Nature 181, 1477.

Margot, P. \& Karamata, D. (1992). Identification of the structural genes for $N$-acetylmuramoyl-L-alanine amidase and its modifier in Bacillus subtilis 168: inactivation of these genes by insertional mutagenesis has no effect on growth or cell separation. Mol Gen Genet 232, 358-366.

Margot, P., Mauel, C. \& Karamata, D. (1994). The gene of the $N$ acetylglucosaminidase, a Bacillus subtilis cell wall hydrolase not involved in vegetative cell autolysis. Mol Microbiol 12, 535-545.

Pooley, H. \& Karamata, D. (1984). Genetic analysis of autolysindeficient and flagellaless mutants of Bacillus subtilis. J Bacteriol 160, 1123-1129.

Rashid, M. H., Kuroda, A. \& Sekiguchi, J. (1993). Bacillus subtilis mutant deficient in the major autolytic amidase and glucosaminidase is impaired in motility. FEMS Microbiol Lett 112, 135-140.

Rashid, M. H., Mori, H. \& Sekiguchi, J. (1995). Glucosaminidase of Bacillus subtilis: cloning, regulation, primary structure and biochemical characterization. Microbiology 141, 2391-2404.

Rogers, H. J., Thurman, P. F. \& Burdett, I. D. J. (1983). The bactericidal action of $\beta$-lactam antibiotics on an autolysindeficient strain of Bacillus subtilis. J Gen Microbiol 129, 465-478.

Rogers, H. J., Taylor, C., Rayter, S. \& Ward, J. B. (1984). Purification and properties of autolytic endo- $\beta$ - $N$-acetylglucosaminidase and the $N$-acetylmuramoyl-L-alanine amidase from Bacillus subtilis strain 168. J Gen Microbiol 130, 2395-2402.

Sambrook, J., Fritsch, E. F. \& Maniatis, T. (1989). Molecular Cloning: a Laboratory Manual, 2nd edn. Cold Spring Harbor, NY: Cold Spring Harbor Laboratory.

Smith, T. J. \& Foster, S. J. (1995). Characterisation of the involvement of two compensatory autolysins in mother cell lysis during sporulation of Bacillus subtilis 168. J Bacteriol 177, 3855-3862.

Smith, T. J., Blackman, S. A. \& Foster, S. J. (1996). Peptidoglycan hydrolases of Bacillus subtilis 168. Microb Drug Resist 2, 113-118.

Steinmetz, M. \& Richter, R. (1994). Plasmids designed to alter the antibiotic resistance expressed by insertion mutations in Bacillus subtilis, through in vivo recombination. Gene 142, 79-83.

Stewart, G. S. A. B., Johnstone, K., Hagelberg, E. \& Ellar, D. J. (1981). Commitment of bacterial spores to germinate. Biochem J 198, 101-106.

Ward, J. B. \& Williamson, R. (1984). Bacterial autolysins: specificity and function. In Microbial Wall Synthesis and Function, pp. 159-166. Edited by C. Nombela. Amsterdam: Elsevier.

Received 24 June 1997; revised 1 September 1997; accepted 12 September 1997. 